\title{
THE FREQUENCY OF FOLLICLE-STIMULATING HORMONE RECEPTOR 2039A>G GENE POLYMORPHISM AND THE RISK OF MALE INFERTILITY IN ALBANIAN POPULATION
}

\author{
Elezaj Shkelzen ${ }^{1}$, Frane Paić ${ }^{2}$, Feodora Stipoljev ${ }^{3}$, Zafer Gashi $^{4}$, Afrim Zeqiraj ${ }^{4}$, \\ Albert Lila ${ }^{5}$ and Tamara Nikuševa Martić ${ }^{2}$ \\ ${ }^{1}$ University of Zagreb, School of Medicine, Zagreb, Croatia; \\ ${ }^{2}$ Laboratory for Epigenetics and Molecular Medicine, Department of Biology and Medical Genetics, \\ School of Medicine, Zagreb, Croatia; \\ ${ }^{3}$ Laboratory for Cytogenetics, Department of Gynecology and Obstetrics, \\ Sveti Duh University Hospital, Zagreb, Croatia; \\ ${ }^{4}$ Department of Biochemistry, FAMA College, Republic of Kosovo; \\ ${ }^{5}$ Department of Gynecology, Regional Hospital, Gjakova, Republic of Kosovo
}

\begin{abstract}
SUMMARY - The purpose of this study was to determine the prevalence of allele and genotype variants of the follicle-stimulating hormone receptor (FSHR) gene polymorphic region at position Asn680Ser in the Albanian male population and associate them with the clinical parameters of infertility. The study included 114 infertile men (mean age 35.04 \pm 5.85 years) stratified according to the level of spermatogenetic impairment (oligoasthenozoospermia, asthenozoospermia and normospermia) and 112 fertile men (mean age 36.44 \pm 7.05 years) with normal semen parameters. Genotyping of the FSHR gene at position 680 was performed by TaqMan genotyping assay. All the participants underwent semen analysis, and serum reproductive hormones (FSH, luteinizing hormone, prolactin and testosterone) were also measured. The FSHR Asn680Ser genotype frequencies were as follows: Asn/Ser 42\%, Ser/Ser 33.9\% and Asn/Asn 24.1\% in the control group, and Asn/Ser 56.1\%, Ser/Ser $22.8 \%$ and $A s n / A s n 21.1 \%$ in the whole group of infertile men ( $\chi^{2}$-test: $\left.P=0.08\right)$. There was no statistically significant correlation between serum hormone levels and semen characteristics or between fertility status and FSHR Asn680Ser gene variants in the control group and the group of infertile men. However, adjusted logistic regression analysis (age, body mass index, smoking and alcohol as covariates) revealed increased odds ratio for male infertility among heterozygous Asn/Ser genotype carriers associated with lower values of semen parameters (normal morphology, concentration, total sperm count and motility). In conclusion, our case-control study further confirmed previous reports on no significant association between the FSHR Asn680Ser polymorphisms and male infertility. Nevertheless, the data presented herein indicate that the Asn/Ser genotype may increase the risk of male infertility in Albanian population.
\end{abstract}

Key words: Infertility, male; Prolactin; Testosterone; Receptors, FSH; Case-control studies

\section{Introduction}

Normal function of cells involved in spermatogenesis, including the production of follicle-stimulating hormone (FSH) by these cells, is dependent on the
Correspondence to: Afrim Zeqiraj, $M S$, Department of Biochemistry, FAMA College, Republic of Kosovo

E-mail: afi_istog@hotmail.com

Received July 9, 2018, accepted July 24, 2018 
FSH receptor $(\mathrm{R})$ gene expression ${ }^{1,2}$. However, the impact of FSHR gene variants including the single nucleotide polymorphisms (SNPs) on male fertility is currently not yet well understood. The human FSHR gene spans a region of $54 \mathrm{~kb}$, consists of ten exons and nine introns, and maps to chromosome 2q. The first nine exons encode the extracellular domain, whereas the transmembrane and the intracellular receptor domains are encoded by exon 10 of the FSHR gene ${ }^{3}$. Mutation screening of the FSHR gene revealed various SNPs both in the promoter region and the coding region ${ }^{4-6}$. The most studied $\mathrm{SNP}$ in the promoter region is located at position c. -29 (c. $-29 \mathrm{G}>\mathrm{A}$, rs1394205), while the two common SNPs located in exon 10 (c.919A $>$ G, p.Thr307Ala, rs6165 and c.2039A $>$ G, p.Asn680Ser, rs6166) are in strong linkage disequilibrium, yielding two distinct allelic variants, Thr307-Asn680 and Ala307-Ser680, with the latter representing approximately $40 \%$ of FSH receptor alleles worldwide ${ }^{4-6}$.

Up to date, several case-control studies and their meta-analyses were conducted to evaluate the association between the FSHR polymorphic regions and male infertility ${ }^{7-12}$. Although mostly inconclusive, some of them revealed significant difference in the distribution of FSHR 2039A $>$ G allelic variants between infertile men and fertile healthy controls, thus showing that ethnic differences could be contributory ${ }^{7,12-14}$. Because no study addressing the effect of FSHR 2039A $>$ G polymorphism on male (in)fertility in Albanian population has been conducted so far, we analyzed the allelic and genotype distribution of this polymorphic region and their association with serum reproductive hormones and semen quality parameters in 114 infertile men and 112 proven fathers from the Dukagin region in the Republic of Kosovo.

\section{Materials and methods}

\section{Study population}

A total of 114 infertile male patients were included in this study. All patients presented with a history of unexplained primary infertility and were stratified into oligozoospermic (sperm count $<15 \times 10^{6}$ spermatozoa per $\mathrm{mL}$ of ejaculate; $\mathrm{n}=67$ ), normoasthenozoospermic (sperm count $\geq 15 \times 10^{6}$ spermatozoa per $\mathrm{mL}$ of ejaculate, progressive motility $\mathrm{PR}(\mathrm{A}+\mathrm{B}) \%<32 ; \mathrm{n}=28)$ and normospermic (normal semen quality; $n=19$ ) infertile men groups according to the World Health Organization criteria (WHO, 2010). Infertility was defined as the inability to conceive despite 24-month unprotected intercourse with the same partner. All patients had a fertile female partner proven by extensive fertility evaluation. Participants with genital tract pathologies, leukocytospermia (more than $10^{6}$ white blood cells per $\mathrm{mL}$ of ejaculate), positive mixed agglutination reaction and $\mathrm{Y}$ chromosome microdeletion or karyotype abnormalities were not included in the study. Control group consisted of 112 proven fertile men (fathered at least one child without assisted reproductive technologies) with normal semen parameters according to the WHO guidelines (WHO, 2010). All patients and controls were ethnic Albanians from the Dukagjin region in the Republic of Kosovo. All participants gave a written informed consent, while the institutional Ethics Committee approved the study protocol in accordance with the Declaration of Helsinki.

\section{Medical evaluations}

Upon inclusion, all participants underwent medical examination, which included a detailed reproductive history, determination of testicular size by ultrasonography, urine analysis and complete blood count, biochemical profile and thyroid function test, measurement of serum FSH, luteinizing hormone (LH), testosterone (T) and prolactin (PRL) levels by BioMérieux Mini Vidas Automated Immunoassay Analyzer (bioMérieux S.A., Marcy l'Etoile, France) and semen analysis according to the $\mathrm{WHO}$ recommendations of 2010. At least two semen samples were provided by each participant after an abstinence period of 3-4 days one month apart.

\section{Genotyping}

Genomic DNA was extracted from peripheral blood leukocytes using the whole blood PureLink ${ }^{\mathrm{TM}}$ Genomic DNA Mini Kit (Invitrogen, Life Technologies Corporation, Carlsbad, CA, USA). The FSHR gene polymorphism at codon 680 (N680S, rs6166. 2039A $>$ G) was analyzed by the TaqMan allelic discrimination assay (Assay ID: C__2676874_10; cat. number 4351376; Lot Number: P130805-002H05; Assay Mix Concentration; 40X; Forward Primer Concentration $36 \mu \mathrm{M}$; Reverse Primer Concentration 36 
$\mu \mathrm{M}$; concentration of forward and reverse primer: 900 $\mathrm{nM}$, probe concentration: $200 \mathrm{nM} \mathrm{FAM}^{\mathrm{TM}} / \mathrm{VIC}^{\circledR}$ dyes; Context Sequence [VIC/FAM]:AGGGACAA GTATGTAAGTGGAACCA[C/T]TGGT GACTCTGGGACTGAAGAGCA (Life Technologies Corporation, Carlsbad, CA, USA) using the Applied Biosystems Real-Time PCR System 7500 (Applied Biosystems, Foster City, CA, USA). Each polymerase chain reaction $(\mathrm{PCR})(25 \mu \mathrm{L})$ contained 1.25 $\mu \mathrm{L}$ TaqMan ${ }^{\circledR}$ SNP Genotyping Assay (X20 dilution), 12.50 $\mu \mathrm{L}$ TaqMan Universal PCR Master Mix II No AmpErase UNG (2X dilution) (Applied Biosystems, Foster City, CA, USA), $5 \mu \mathrm{L}$ of genomic DNA and $6.25 \mu \mathrm{L}$ of DNase/RNase free water. PCR was carried out in two steps: absolute quantification and allelic discrimination. For absolute quantification, the cycles were as follows: AmpliTaq Gold Enzyme activation at $95^{\circ} \mathrm{C}$ for $10 \mathrm{~min}(1$ cycle); 40 cycles: denaturation at $92{ }^{\circ} \mathrm{C}$ for $15 \mathrm{~s}$, and annealing/extension at $60{ }^{\circ} \mathrm{C}$ for $1 \mathrm{~min}$. The results were verified by Applied Biosystems Real-Time PCR System 7500 allelic discrimination software (Applied Biosystems, Foster City, CA, USA).

\section{Statistical analysis}

Mean, standard deviation, median and $5-95^{\text {th }}$ percentiles were calculated for general characteristics [age, body mass index (BMI)] and main outcome variables (hormonal and seminal variables, total testis volume) using SPSS software version 22 (SPSS Inc., Chicago, IL, USA). Before statistical analysis, data were tested for normal distribution using the KolmogorovSmirnov and Shapiro-Wilk test. For data not normally distributed, the Kruskal-Wallis test was applied. Hardy-Weinberg equilibrium was assessed by $\chi^{2}$ analysis. Odds ratio (OR) and $95 \%$ confidence interval (95\% CI) were calculated to measure the strength of the association, where applicable. Logistic regression analysis was used to adjust statistical findings for confounding factors (age, BMI, smoking and alcohol consumption). Clinical data were compared among different genotypes by one-way ANOVA and $\chi^{2}$-test, where appropriate. Genotype association tests were performed under co-dominant, dominant, recessive and over-dominant genetic models. A nominal level of significance $\mathrm{P}<0.05$ was accepted and corrected according to Bonferroni procedure (the corrected level of significance is: $\mathrm{P}_{c}=0.05 / \mathrm{N} ; \mathrm{N}$, number of independent comparisons). All reported $\mathrm{P}$ values were two-sided and uncorrected unless stated otherwise.

\section{Results}

\section{Demographic and clinical characteristics of study participants}

Table 1 shows the mean and median values of demographic and clinical parameters in infertile men and control group. As can be seen, no statistically significant differences were found in age (control: $36.44 \pm 7.047$ years; infertile men group: $35.04 \pm 5.854$ years), BMI and alcohol consumption between the control and infertile men groups after Bonferroni correction for multiple testing was applied [one-way ANOVA/independent sample $t$ test; Kruskal Wallis / Mann-Whitney U test $\mathrm{Pc}=0.05 / 55=9.1 \times 10^{-4}$; taking into account the number of independent measurements (eleven) and tested study samples (five)]. However, the number of ever smokers was statistically higher in overall $(\mathrm{P}=0.0001)$, normoasthenozoospermic $(\mathrm{P}=0.001)$ and normospermic $(\mathrm{P}=0.004)$ infertile men subgroups relative to control participants $\left[\chi^{2}\right.$-test $\mathrm{Pc}_{\mathrm{C}}=0.05 / 10=0.005$; taking into account the number of independent measurements (two) and tested study samples (five)].

\section{Genotype distribution and allele frequencies}

The results of statistical analysis of the distribution of Asn680Ser FSHR gene polymorphism genotype and allele frequencies between infertile men $(n=114)$ and controls $(\mathrm{n}=112)$ are shown in Table 2 . The allele frequencies were $54.91 \% \operatorname{Ser}(\mathrm{S})$ and $45.09 \%$ Asn $(\mathrm{N})$ in the control group, and $50.88 \%$ Ser and $49.12 \%$ Asn in the overall infertile men group $\left(\mathrm{P}=0.39, \chi^{2}\right.$-test $)$. No significant differences in allele distribution relative to control were observed for any of the infertility subgroups (i.e. oligoastheno-, normoastheno- and normospermia) analyzed in the study. In addition, no significant difference was observed in genotype distribution between control and overall infertile men group, and the genotypes were in Hardy-Weinberg equilibrium in both groups (infertile men group: $\chi^{2}=1.73, \mathrm{df}=1$, $\mathrm{P}=0.19$; control group: $\chi^{2}=2.61, \mathrm{df}=1, \mathrm{P}=0.11$ ). Furthermore, the significant difference in genotype distri- 


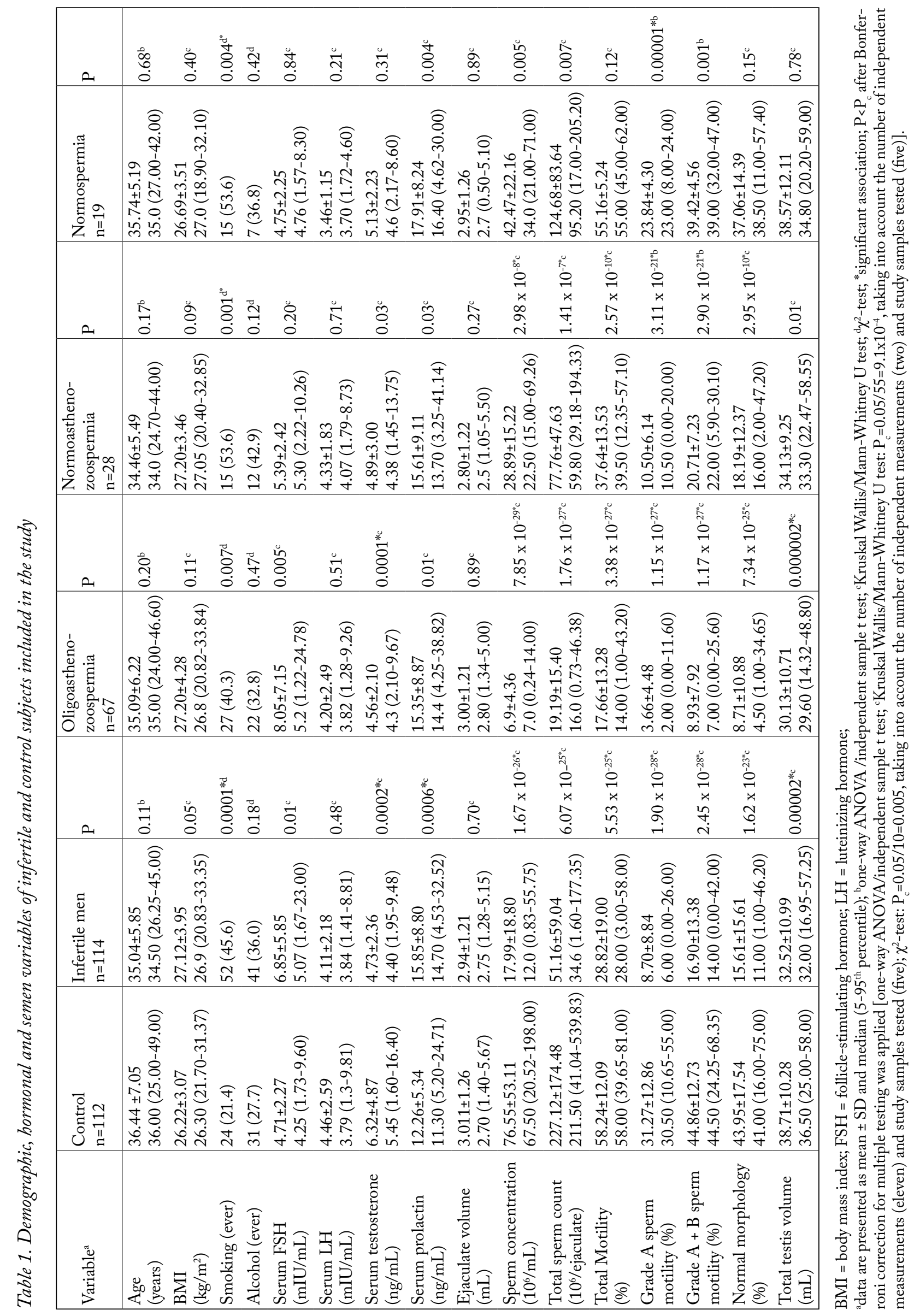




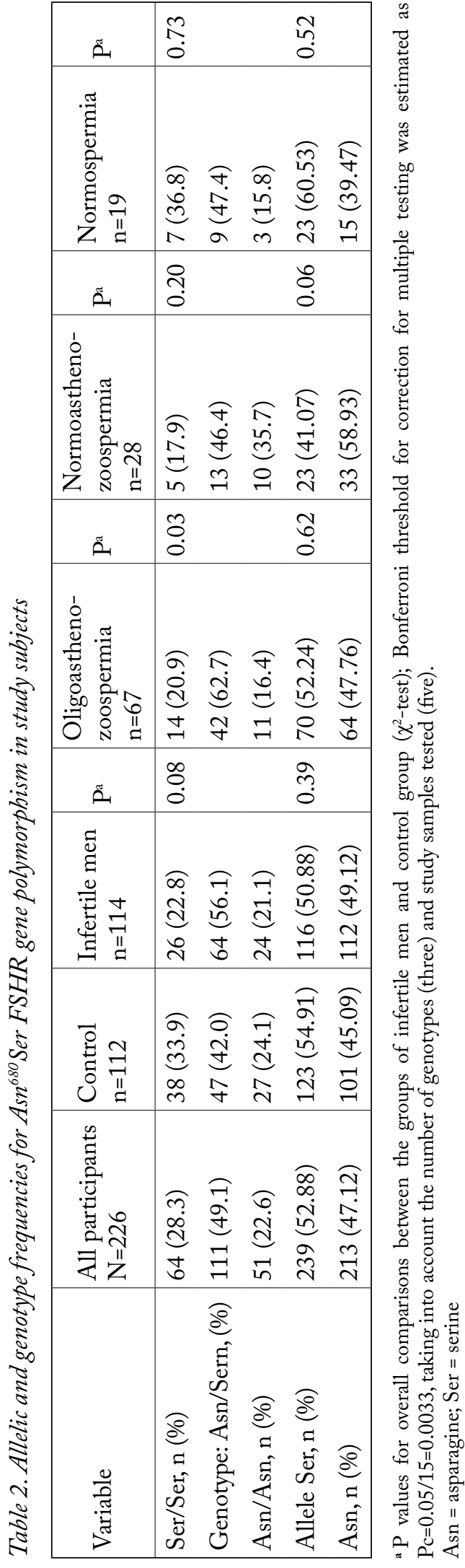

bution between the oligoazoospermic infertile group and control group was lost when Bonferroni correction for multiple testing was applied.

\section{Association of N680S FSHR gene polymorphism with infertility}

Table 3 shows the results of contingency table $\left(\chi^{2}-\right.$ test) analysis of Asn680Ser FSHR genotype and allele distribution among the studied groups. As can be seen, OR between the Asn and Ser allele for development of infertility was higher in the overall, as well as in the oligo- and normoasthenozoospermic infertile men groups relative to control group, thus indicating the Ser allele as being protective against infertility. However, the effect did not reach statistical significance after Bonferroni correction for multiple testing was applied.

Crude and adjusted (age, BMI, smoking and alcohol as covariates) logistic regression analysis revealed an increased risk of infertility in heterozygous Asn/Ser genotype carriers in the overall $\left(\mathrm{OR}_{\mathrm{A}}=2.36 ; 95 \% \mathrm{CI}\right.$ : 1.19-4.67) and oligoasthenozoospermic $\left(\mathrm{OR}_{\mathrm{A}}=2.745\right.$; 95\% CI: 1.24-6.07) infertile men groups when compared with Ser/Ser genotype carriers. Again, no significant association was observed after Bonferroni correction for multiple testing was applied. Interestingly, relative to Ser/Ser genotype carriers, the homozygous Asn/Asn genotype carriers in the normoasthenozoospermic infertile men group exhibited higher $\left(\mathrm{OR}_{\mathrm{A}}=3.28 ; 95 \% \mathrm{CI}: 0.87-12.30\right)$ nonsignificant risk of infertility compared to Asn/Ser $\left(\mathrm{OR}_{\mathrm{A}}=2.55 ; 95 \%\right.$ CI: 0.76-8.56) genotype carriers. No genetic associations between FSHR Asn680Ser polymorphism and tested reproductive parameters reached Bonferronicorrected statistical significance level irrespective of whether the groups were analyzed under the co-dominant, dominant, recessive or over-dominant genetic model $[\mathrm{Pc}=0.05 / 20=0.0025$, taking into account the number of studied groups (five) and tested genotype models (four)]. Nevertheless, the best-fitting overdominant genetic model (Asn/Ser vs. Ser/Ser + Asn/ Asn) further implicated an increased risk of male infertility in heterozygous Asn/Ser genotype carriers either in the overall infertile men group $(\mathrm{ORA}=2.032$; 95\% CI: $1.138-3.626)$ or when subgrouping them according to various degrees of spermatogenetic impairment (Table 4). 
Table 3. Contingency table analysis for Asn680Ser FSHR genotype and allele distribution between the subgroups of infertile men and control group

\begin{tabular}{|c|c|c|c|}
\hline Variable & $\begin{array}{l}\text { Frequency } \\
\mathrm{n}(\%)\end{array}$ & $\begin{array}{l}\text { Contingency tables } \\
\text { OR }(95 \% \mathrm{CI})\end{array}$ & $\mathrm{P}^{\mathrm{a}}$ \\
\hline $\begin{array}{l}\text { Infertile men }(\mathrm{N}=114) \\
\text { Ser/Ser }\end{array}$ & $26(22.8)$ & $0.5754(0.3199-1.0347)$ & 0.06 \\
\hline Asn /Ser & $64(56.1)$ & $1.7702(1.0451-2.9984)$ & 0.03 \\
\hline Asn/Asn & $24(21.1)$ & $0.8395(0.4495-1.5679)$ & 0.58 \\
\hline Ser & $116(50.88)$ & $0.8505(0.5876-1,2309)$ & 0.39 \\
\hline Asn & $112(49.12)$ & $1.1758(0.8124-1.7018)$ & \\
\hline $\begin{array}{l}\text { Oligoasthenozoospermia }(\mathrm{n}=67) \\
\text { Ser/Ser }\end{array}$ & $14(20.9)$ & $0.5144(0.2537-1.0432)$ & 0.063 \\
\hline Asn /Ser & $42(62.79$ & $2.3234(1.2483-4.3245)$ & 0.007 \\
\hline Asn/Asn & $11(16.4)$ & $0.6184(0.284-1.3463)$ & 0.22 \\
\hline Ser & $70(52.24)$ & $0.8981(0.5847-1.3795)$ & 0.62 \\
\hline Asn & $64(47.76)$ & $1.1134(0.7249-1.7102)$ & \\
\hline $\begin{array}{l}\text { Normoasthenozoospermia }(\mathrm{n}=28) \\
\text { Ser/Ser }\end{array}$ & $5(17.9)$ & $0.4233(0.1491-1.2016)$ & 0.10 \\
\hline Asn /Ser & $13(46.4)$ & $1.1986(0.5215-2.7546)$ & 0.67 \\
\hline Asn/Asn & $10(35.7)$ & $1.749(0.7211-4.2421)$ & 0.21 \\
\hline Ser & $23(41.07)$ & $0.5723(0.316-1.0365)$ & 0.06 \\
\hline Asn & $33(58.93)$ & $1.7473(0.9648-3.1644)$ & \\
\hline $\begin{array}{l}\text { Normospermia }(\mathrm{n}=19) \\
\text { Ser/Ser }\end{array}$ & $7(36.8)$ & $1.136(0.4134-3.1217)$ & 0.81 \\
\hline Asn /Ser & $9(47.4)$ & $1.2447(0.4692-3.3019)$ & 0.66 \\
\hline Asn/Asn & $3(15.8)$ & $0.5903(0.1598-2.181)$ & 0.43 \\
\hline Ser & $23(60.53)$ & $1.2591(0.6242-2.5398)$ & 0.52 \\
\hline Asn & $15(39.47)$ & $0.7942(0.3937-1.6021)$ & \\
\hline
\end{tabular}

$\mathrm{OR}=$ odds ratio with $95 \% \mathrm{CI} ; 95 \%=95 \%$ confidence interval; ${ }^{\text {a }} \mathrm{P}$ values for individual genotype and allele comparisons between the subgroups of infertile men and control group; Bonferroni threshold for correction for multiple testing was estimated as $\mathrm{Pc}=0.05 / 12=0.004$, taking into account the number of genotypes (three) and study samples tested (four). Note: data for control group are not shown.

Asn = asparagine; Ser = serine

\section{Association of N680S FSHR gene polymorphism and reproductive hormones}

Serum FSH level in the infertile men group (6.85 \pm $5.85)$ was higher relative to control group $(4.71 \pm 2.27)$ but the difference did not reach statistical significance after Bonferroni correction for multiple testing was applied $\left(\mathrm{P}=0.01<\mathrm{Pc}=0.05 / 55=9.1 \times 10^{-4}\right)$. Similar results were obtained in the oligoasthenozoospermic, normoasthenozoospermic and normospermic infertile men subgroups compared to control group. Contrary, serum prolactin level was significantly $(\mathrm{P}=0.0006)$ higher in the overall infertile men group compared to control group $(15.85 \pm 8.80$ and $12.26 \pm 5.34$, respectively). However, the difference did not reach statistical significance in any of the infertile men subgroups analyzed. The overall infertile men group also showed lower LH (4.11 \pm 2.18 and $4.46 \pm 2.59$, respectively) and testosterone $(4.73 \pm 2.36$ and $12.26 \pm 5.34$, respectively) serum levels relative to control group. Again, the differences did not reach statistical significance $\left(\mathrm{P}=0.48<\mathrm{Pc}=0.05 / 55=9.1 \times 10^{-4}\right)$ for $\mathrm{LH}$ levels between these two groups or between any other infertile men subgroup and control group, while testosterone exhibited a significantly lower serum level only in the 
Table 4. Risk of infertility and Asn680Ser FSHR gene polymorphism among infertile men included in the study

\begin{tabular}{|c|c|c|c|c|c|c|}
\hline Variable & $\begin{array}{l}\text { Control n } \\
(\%)\end{array}$ & $\begin{array}{l}\text { Case } \\
\text { n (\%) }\end{array}$ & $\begin{array}{l}\text { Logistic regression } \\
\text { Crude OR }(95 \% \\
\text { CI) }\end{array}$ & $\mathrm{P}$ & $\begin{array}{l}\text { Logistic regression } \\
\mathrm{OR}_{\mathrm{A}}(95 \% \mathrm{CI})\end{array}$ & $\mathrm{P}$ \\
\hline \multicolumn{7}{|l|}{ Infertile men (N=114) } \\
\hline Ser/Ser & $38(33.9)$ & $26(22.8)$ & 1.0 (Reference) & $0.084^{\mathrm{a}}$ & 1.0 (Reference) & $0.042^{\mathrm{a}}$ \\
\hline Co-dominant Asn/Ser & $47(42.0)$ & $64(56.1)$ & $1.99(1.07-3.72)$ & $0.03^{\mathrm{b}}$ & $2.36(1.19-4.67)$ & $0.01^{\mathrm{b}}$ \\
\hline Asn/Asn & $27(24.1)$ & $24(21.1)$ & $1.30(0.62-2.73)$ & $0.49^{\mathrm{b}}$ & $1.40(0.62-3.15)$ & $0.42^{\mathrm{b}}$ \\
\hline Dominant Ser/Ser & $38(33.9)$ & $26(22.8)$ & 1.0 (Reference) & & 1.0 (Reference) & \\
\hline Asn/Asn + Asn/Ser & $74(66.1)$ & $88(77.2)$ & $1.74(0.97-3.13)$ & $0.065^{\mathrm{b}}$ & $1.99(1.05-3.80)$ & 0.035 \\
\hline Recessive Ser/Ser +Asn/Ser & $85(75.9)$ & $90(79)$ & 1.0 (Reference) & & 1.0 (Reference) & \\
\hline Asn/Asn & $27(24.1)$ & $24(21.1)$ & $0.84(0.45-1.57)$ & $0.583^{\mathrm{b}}$ & $0.81(0.41-1.60)$ & $0.548^{\mathrm{b}}$ \\
\hline Over-dominant Ser/Ser + Asn/Asn & $65(58.0)$ & $50(43.9)$ & 1.0 (Reference) & & 1.0 (Reference) & \\
\hline Asn/Ser & $47(42.0)$ & $64(56.1)$ & $1.77(1.05$ & $0.034^{\mathrm{b}}$ & 2.03 & $0.016^{\mathrm{b}}$ \\
\hline \multicolumn{7}{|l|}{ Oligoasthenozoospermia $(\mathrm{n}=67)$} \\
\hline Ser/Ser & $38(33.9)$ & $14(20.9)$ & 1.0 (Reference) & $0.03^{\mathrm{a}}$ & 1.0 (Reference) & $0.02^{\mathrm{a}}$ \\
\hline Co-dominant Asn/Ser & 47( & $42(62.79$ & 2.43 & $0.02^{\mathrm{b}}$ & 2.7 & $0.01^{\mathrm{b}}$ \\
\hline Asn/Asn & $27(24.1)$ & $11(16.4)$ & 1.11 & $0.83^{b}$ & 1.18 & $0.75^{\mathrm{b}}$ \\
\hline Dominant Ser/Ser & $38(33.9)$ & $14(20.9)$ & 1.0 (Reference) & & 1.0 (Reference) & \\
\hline Asn/Asn & $74(66.1)$ & $53(79.1)$ & $1.94(0.96-3.94)$ & $0.065^{\mathrm{b}}$ & $2.18(1.02-4.65)$ & $0.045^{\mathrm{b}}$ \\
\hline er/Ser + Asn/Ser & $85(75.9)$ & $56(83.6)$ & 1.0 (Reference) & & 1.0 (Reference) & \\
\hline Asn/Asn & $27(24.1)$ & $11(16.4)$ & $0.62(0.28-1.35)$ & $0.226^{\mathrm{b}}$ & $0.60(0.26-1.37)$ & $0.224^{\mathrm{b}}$ \\
\hline Over-dominant Ser/Ser + Asn/Asn & $65(58.0)$ & $25(37.3)$ & 1.0 (Reference) & & 1.0 (Reference) & \\
\hline Asn/Ser & $47(42.0)$ & $42(62.7)$ & $2.32(1$ & $0.008^{\mathrm{b}}$ & $2.56(1$ & $0.006^{\mathrm{b}}$ \\
\hline \multicolumn{7}{|l|}{ Normoasthenozoospermia $(\mathrm{n}=28)$} \\
\hline Co-dominant Asn/Ser & $47(42.0)$ & & 2.10 & $0.19^{\mathrm{b}}$ & & $0.13^{\mathrm{b}}$ \\
\hline Asn/Asn & $27(24.1)$ & $10(35.7)$ & $2.82(0.86-9.17)$ & $0.09^{\mathrm{b}}$ & $3.28(0.87-12.30)$ & $0.08^{\mathrm{b}}$ \\
\hline Dominant $\mathrm{Se}$ & $38(33.9)$ & $5(17.9)$ & 1.0 (Reference) & & 1.0 (Reference) & \\
\hline Asn/Asn & $74(66.1)$ & $23(82.1)$ & $2.36(0.83-6.71)$ & 0.106 & $2.80(0.89-8.75)$ & $0.077^{\mathrm{b}}$ \\
\hline Recessive Ser/Ser +Asn/Ser & $85(75.9)$ & $18(64.3)$ & 1.0 (Reference) & & 1.0 (Reference) & \\
\hline Asn/Asn & $27(24.1)$ & $10(35.79$ & $1.75(0.72-4.24)$ & $0.216^{\mathrm{b}}$ & $1.79(0.66-4.89)$ & $0.257^{\mathrm{b}}$ \\
\hline Over-dominant Ser/Ser + Asn/Asn & $65(58.0)$ & $15(53.6)$ & 1.0 (Reference) & & 1.0 (Reference) & \\
\hline Asn/Ser & $47(42.0)$ & $13(46.4)$ & $1.20(0.52-2.76$ & $0.670^{\mathrm{b}}$ & & $0.506^{\mathrm{b}}$ \\
\hline \multicolumn{7}{|l|}{ Normospermia $(n=19)$} \\
\hline & $38(33.9)$ & $7(36.8)$ & $1.0(\mathrm{~F}$ & $0.73^{\mathrm{a}}$ & 1.0 & $0.58^{\mathrm{a}}$ \\
\hline Co-dominant Asn/Ser & $47(42.0)$ & $9(47.4)$ & $1.04(0.35-3.05)$ & $0.94^{b}$ & $1.15(0.35-3.84)$ & $0.82^{\mathrm{b}}$ \\
\hline Asn/Asn & $27(24.1)$ & $3(15.8)$ & $0.60(0.14-2.55)$ & $0.49^{\mathrm{b}}$ & $0.53(0.12-2.43)$ & $0.41^{\mathrm{b}}$ \\
\hline Dominant & $38(33.9)$ & $7(36.8)$ & 1.0 (Reference) & & 1.0 (Reference) & \\
\hline Asn/Asn + Asn/Ser & $74(66.1)$ & $12(63.2)$ & $0.88(0.32-2.42)$ & $0.805^{b}$ & $0.88(0.29-2.68)$ & $0.822^{\mathrm{b}}$ \\
\hline Recessive Ser/Ser +Asn/Ser & $85(75.9)$ & $16(84.2)$ & 1.0 (Reference) & & 1.0 (Reference) & \\
\hline Asn/Asn & $27(24.1)$ & $3(15.8)$ & $0.59(0.16-2.18)$ & $0.429^{\mathrm{b}}$ & $0.49(0.12-1.92)$ & $0.305^{\mathrm{b}}$ \\
\hline Over-dom & $65(58.0)$ & $10(52.6)$ & 1.0 (Reference) & & 1.0 (Reference) & \\
\hline Asn/Ser & $47(42.0)$ & $9(47.4)$ & $1.25(0.47-3.30)$ & $0.660^{\mathrm{b}}$ & $1.48(0.51-4.33)$ & $0.470^{\mathrm{b}}$ \\
\hline
\end{tabular}

$\mathrm{OR}_{\mathrm{A}}=$ adjusted odds ratio (adjusted for age, body mass index, alcohol and smoking status) with 95\% confidence interval (95\% CI); ${ }^{\mathrm{P}} \mathrm{Palues}$ for overall genotype comparisons between the subgroups of infertile men and control group; ${ }^{\mathrm{b}} \mathrm{P}$ values for individual comparisons with reference genotype between the subgroups of infertile men and control group; Bonferroni threshold for correction for multiple testing was estimated as $\mathrm{P}_{c}=0.0025\left[\mathrm{P}_{\mathrm{c}}=0.05 / 20=0.0025\right.$, taking into account the number of study groups (five) and genotype models tested (four) $]$. Note: data for the subgroups of infertile men not shown.

Asn = asparagine; Ser = serine 


\begin{tabular}{|c|c|c|c|c|c|c|c|c|c|c|c|c|c|c|c|}
\hline & $\hat{D}_{1}$ & $\stackrel{n}{0}$ & లె & $\stackrel{\infty}{\circ}$ & $\stackrel{n}{n}$ & 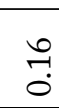 & $\stackrel{2}{8}$ & 8 & 5 & & $\stackrel{\circ}{0}$ & $\begin{array}{l}0 \\
\stackrel{1}{0} \\
0 \\
0\end{array}$ & 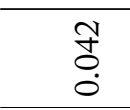 & స̆ & 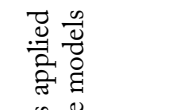 \\
\hline 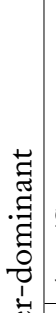 & 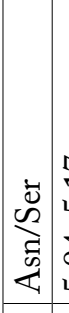 & 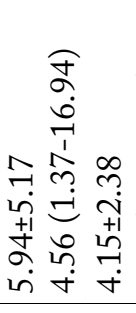 & 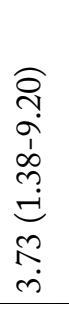 & 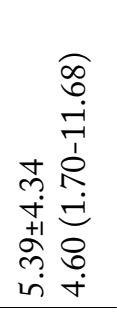 & 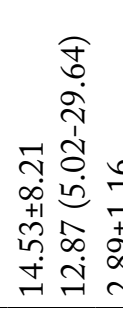 & 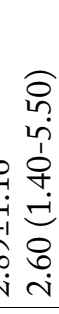 & 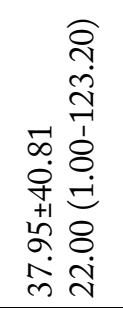 & 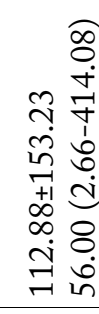 & 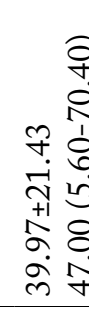 & & $\begin{array}{l}\text { of } \\
+ \\
0 \\
+ \\
\dot{1} \\
0 \\
\dot{0} \\
8 \\
8 \\
\dot{0} \\
\end{array}$ & 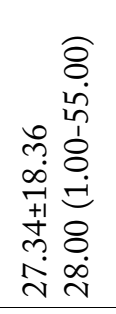 & 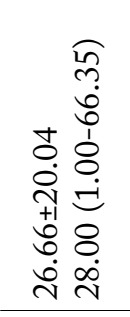 & 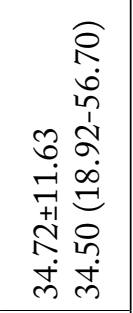 & 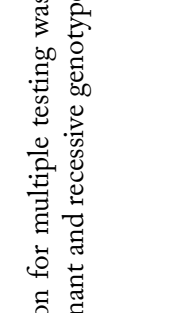 \\
\hline ठे. & 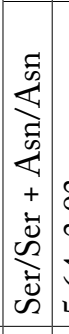 & 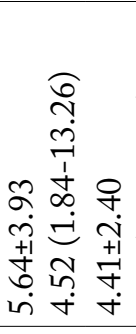 & 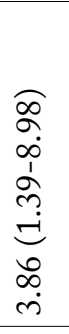 & 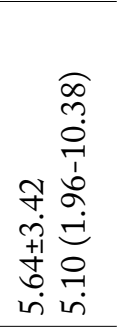 & 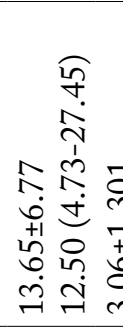 & 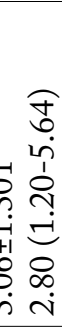 & 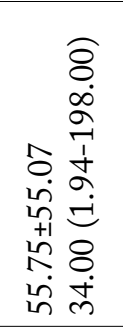 & 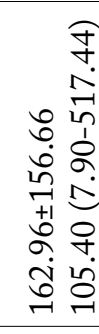 & 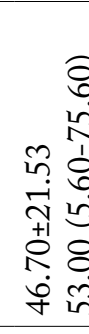 & & 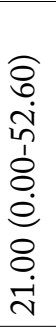 & 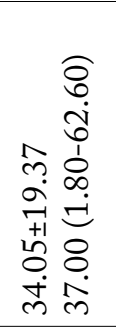 & 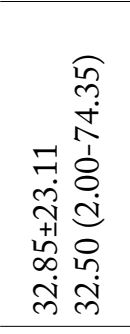 & 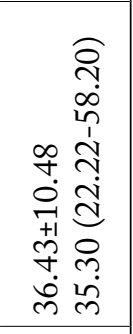 & 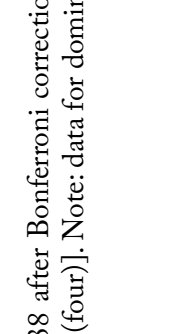 \\
\hline & $\hat{A}_{1}$ & $\stackrel{\infty}{+}$ & $\stackrel{\infty}{+}$ & ت্ণ & ก? & $=$ & $\stackrel{\text { I }}{\circ}$ & ঃ & $\begin{array}{l}2 \\
0 \\
0\end{array}$ & & 今ิ & 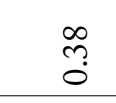 & ஓి & 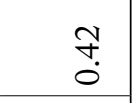 & $\ddot{0}$ \\
\hline & 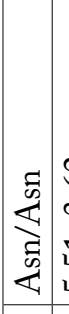 & 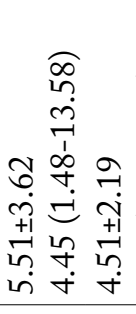 & 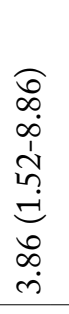 & 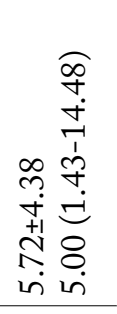 & 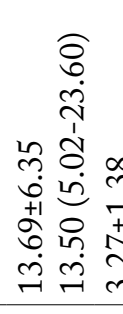 & 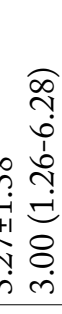 & 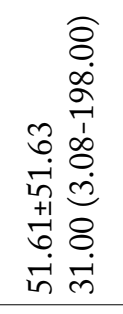 & 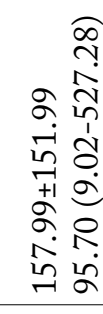 & 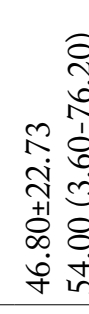 & & 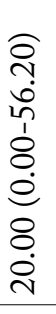 & 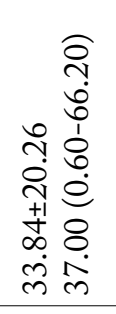 & 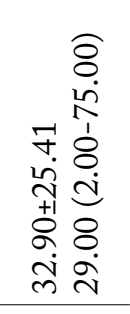 & 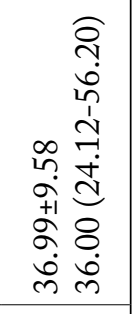 & 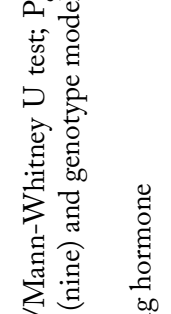 \\
\hline$\vec{\Xi}$ & $\hat{\imath}_{1}$ & กิ่ & $\begin{array}{l}10 \\
0 \\
0\end{array}$ & $\begin{array}{l} \pm \\
0\end{array}$ & กี? & $\begin{array}{l}\text { Jै } \\
0\end{array}$ & ¿̊. & 5 & 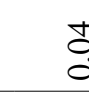 & & & Oे. & 今. & กิ่ & \\
\hline 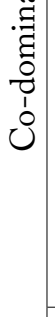 & 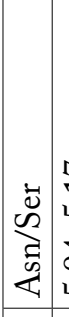 & 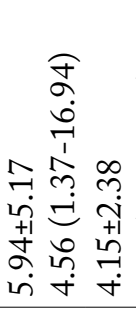 & 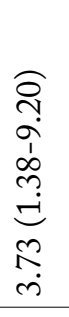 & 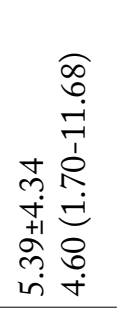 & 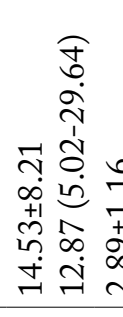 & 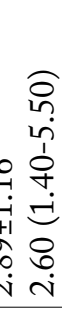 & 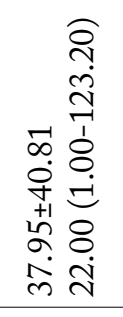 & 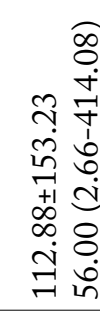 & 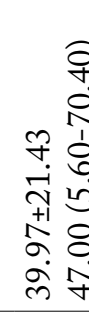 & & 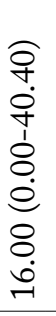 & 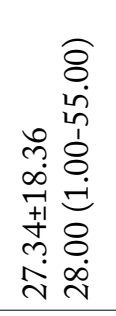 & 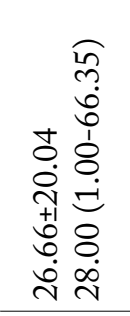 & 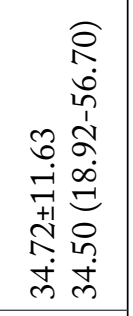 & 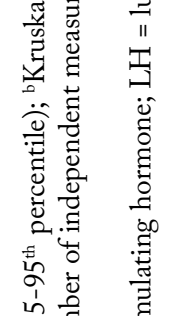 \\
\hline & $\begin{array}{c}\dot{u} \\
\tilde{n} \\
\dot{u} \\
\tilde{n}\end{array}$ & 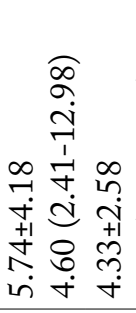 & 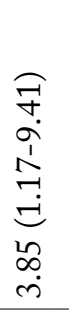 & 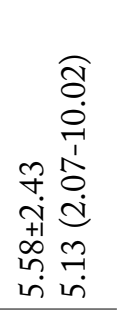 & 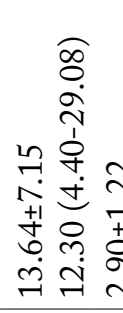 & 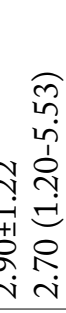 & 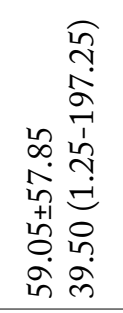 & 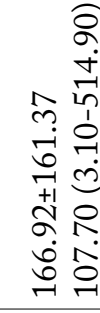 & 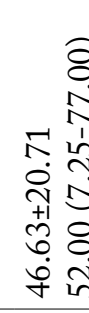 & & 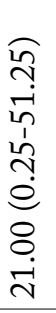 & 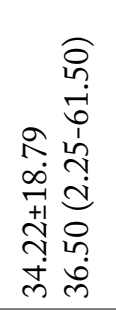 & 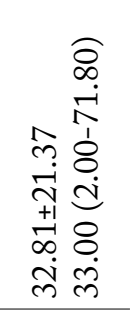 & 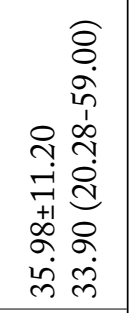 & 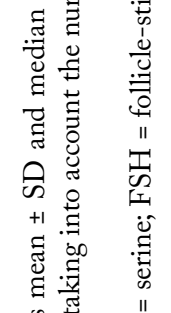 \\
\hline & & 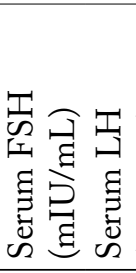 & $\frac{\Xi}{\Xi}$ & 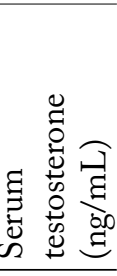 & 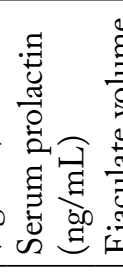 & & 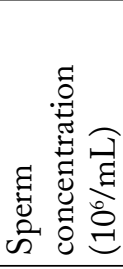 & 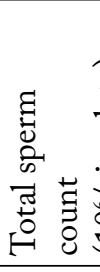 & 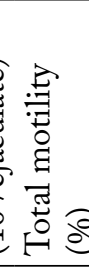 & & & 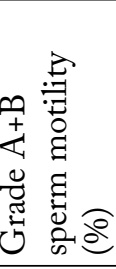 & 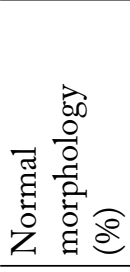 & 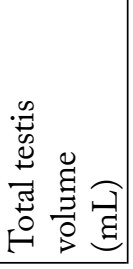 & 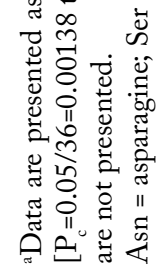 \\
\hline
\end{tabular}


overall $(\mathrm{P}=0.0002)$ and oligoasthenozoospermic $(\mathrm{P}=$ $0.0001)$ infertile men groups relative to control group (Table 1).

Regarding the effect of N680S FSHR polymorphism, we did not observe any statistically significant association of FSHR genotype frequencies with FSH serum levels in either control or any infertile men group analyzed (data not shown). Likewise, no significant differences were found in $\mathrm{LH}$, testosterone and prolactin hormone levels between men with different genotypes (Table 5).

\section{The effects of N680S FSHR gene polymorphism on semen and testicular parameters}

As expected, semen parameters (normal morphology, concentration, total sperm count and progressive motility grade $A$ and grade $A+B$ ) were significantly lower in the overall group, as well as in the oligo- and normo-asthenozoospermic infertile men subgroups when compared to controls. Notably, the progressive sperm motility grade A was also significantly lower in the normospermic infertile men group compared to control group. In addition, total testis volume showed significantly lower values in the overall group and oligoasthenozoospermic infertile men subgroup as compared to control group (Table 1).

We also analyzed the effect of N680S FSHR polymorphism on major semen characteristics (sperm concentration, total sperm count, ejaculate volume, morphology, total and progressive sperm motility grade $\mathrm{A}$ and grade $\mathrm{A}+\mathrm{B}$ ) and total testis volume. For that purpose, data on testicular and seminal parameters in all study groups were pooled together to find variations among the different genotype models analyzed (Table 5). As shown in Table 5, the co-dominant and overdominant genetic models once again implicated an increased risk of male infertility among the Asn/Ser (vs. Ser/Ser or Ser/Ser + Asn/Asn, respectively) genotype carriers regarding their lower values of major sperm parameters (i.e. percentage of cells with normal morphology, total sperm count and concentration, and grade A progressive and total mobility) analyzed (data for dominant and recessive genotype model are not shown). However, none of these associations remained significant after Bonferroni correction for multiple testing was applied $\left[\mathrm{P}_{\mathrm{c}}=0.05 / 36=0.00138\right.$; taking into account the number of independent measurements (nine) and genotype models tested (four)].

\section{Discussion}

In the present study, infertile men and proven fertile control subjects were genotyped to explore the association between the N680S ( $r$ s6166 SNP;2039A>G) FSHR gene polymorphism and male infertility risk in Albanian population from the Dukagjin region in the Republic of Kosovo. To the best of our knowledge, this was the first study examining the effect of FSHR gene polymorphism on male infertility parameters in Albanian population. We found no significant difference in the distribution of N680S genotypes between the overall group of infertile men or oligoastheno-, normoastheno- and normospermic infertile subgroups and the control group of fertile men. Our data agreed with those reported in the previous similar studies conducted in other ethnicities but there are differences in the proportions of genotype and allele distribution between our and others studies $1,6,7,12,15,16$. Namely, our study revealed the dominant frequency distribution of the Asn/Ser genotype to be consistent with its highest rates found in other ethnic groups $\mathrm{s}^{1,6,7,12,15,16}$. However, contrary to other ethnic populations examined so far, the Ser/Ser variant in our population was represented at a higher frequency compared to Asn/Asn homozygotes. Similar results have also been reported previously by our group concerning distribution of the FSHR ASn680Ser genotype variants in Albanian women from the same region ${ }^{17}$. The sociological and cultural features of the Albanian population, which are manifested by the low incidence of marital union with members of other ethnic groups, thus probably preserving the genetic 'pool' originating from the indigenous population, may be responsible for this effect.

As a complex hormone-regulated disorder, male infertility is regulated by the hypothalamus-pituitarygonad axis with FSH, LH and testosterone as the prime regulators of germ cell development ${ }^{18,19}$. In our study, we found higher FSH and prolactin and lower $\mathrm{LH}$ and testosterone serum levels in the infertile men than in the control group men, but the difference reached statistical significance only for testosterone and prolactin serum levels in the overall group of infertile men, and for testosterone levels in the oligoasthenozoospermic subgroup compared to control group.

In the infertile men, a higher concentration of FSH is usually a reliable indicator of severe germinal epithelial damage, and was shown to be associated with azo- 
ospermia and severe oligozoospermia ${ }^{20,21}$. Likewise, low levels of serum LH and testosterone are found in men with oligoasthenozoospermia while hyperprolactinemia causes infertility in around $11 \%$ of oligospermic males ${ }^{22-25}$. Previous reports suggest that differences in the genotype and allele frequency of Asn680Ser FSHR polymorphic region might represent an important genetic factor contributing to phenotypic expression of essential fertility parameters. This was clearly demonstrated for their influence on basal serum FSH concentrations in women, length and dynamics of menstrual cycle and effective FSH amount during ovarian stimulation for in vitro fertility techniques ${ }^{4,16}$. For example, women with the Ser/Ser receptor variant seem to be less sensitive to FSH stimulation and have significantly higher serum FSH levels in the follicular phase of the menstrual cycle than women with the Asn/Asn isoform ${ }^{26}$. The common FSHR polymorphisms in exon 10 and the promoter of FSHR gene have also been extensively studied in male infertility ${ }^{16}$. However, so far, there is no clear association of male infertility with the ASn680Ser FSHR polymorphic region. Most of the reports suggest no significant differences in carrier frequencies of individual FSHR Asn680Ser polymorphic variants between infertile and fertile men from various ethnic groups ${ }^{6,8,9,27-36}$. The same results were obtained in a meta-analysis of these studies $^{8-11}$. Similarly, the effect of Asn680Ser FSHR isoforms on clinical reproductive parameters such as FSH level, sperm count and total testis volume also failed to show any consistent associations ${ }^{16,28-30,33,34,37,38}$.

Like most of the previous reports, our study showed no statistically significant association of Asn680Ser FSHR genotype and allelic variants with serum FSH, $\mathrm{LH}$, testosterone and prolactin levels in control and infertile men either. However, we did notice the increased although nonsignificant risk of male infertility among heterozygous Asn/Ser (vs. Ser/Ser or Ser/Ser + Asn/Asn, respectively) genotype carriers regarding their lower values of major sperm parameters (total sperm count, concentration, morphology and motility) both in the overall group of infertile men and the infertility subgroups based on various degrees of spermatogenic impairment.

A slight influence of the ASn680Ser FSHR gene polymorphism on male reproductive parameters has also been demonstrated recently by Grigorova et al. ${ }^{12}$. They detected significant association between the
FSHR Ser680 allele and lower total testis volume in both the Baltic cohort of men and Estonian idiopathic infertility group ${ }^{12}$. Subsequent meta-analysis further supported their findings showing a moderate nonsignificant effect between the FSHR Ser680 variant and higher serum FSH level, and significantly lower total testosterone levels. However, no statistically significant associations were identified with serum LH levels or with any of the sperm parameters ${ }^{12}$. A previous study in infertile men from Egyptian population reported by Zalata et al. also showed significantly higher serum FSH (Ser/Ser vs. Asn/Asn) and lower total serum testosterone (Asn/Ser and Ser/Ser vs. Asn/Asn) in Ser680 variant carriers $^{13}$. They also detected a nonsignificant decrease in sperm concentration, grade $A$ and grade $\mathrm{A}+\mathrm{B}$ progressive motility, and normal sperm morphology in Ser/Ser and Asn/Ser compared with the Asn/Asn genotype form ${ }^{13}$. Furthermore, Tsitlakidis et al. have recently reported on a significant association of heterozygous Asn/Ser genotype form with different size of the right testis in infertile Greek men but no association of any genotype form with hormonal serum levels (FSH, LH, testosterone, and prolactin) or sperm parameters (semen volume, sperm count, morphology and motility) was detected ${ }^{14}$. In addition, a recent meta-analysis reported by $\mathrm{Wu}$ et al. detected association of Asn680Ser FSHR Ser/Ser genotype form (Ser/Ser vs. Asn/Asn and Ser/Ser vs. Asn/Asn + Asn/Ser) with an increased risk of male infertility ${ }^{7}$ One of the main reasons for the observed differences between their results and the findings of our study regarding the overall influence of the ASn680Ser FSHR gene polymorphism on infertility status and identification of effective genotype responsible for modification of sperm parameters can be found in population stratification and ethnic and geographic variation, but additional genetic and environmental factors cannot be excluded. Since the phenotypic effects of individual polymorphism may be modulated by the overall genetic background, a combined effect of other polymorphic regions and ASn680Ser allele and genotype variants on male infertility should also be examined.

For example, $\mathrm{Wu}$ et al. report that homozygous combination of FSHR gene polymorphism at codon 307 and 680 (i.e. Thr/Thr + Asn/Asn) may be responsible for male infertility in patients from north Chi$\mathrm{na}^{11}$. In addition, Lindgren et al. report that Thr307- 
Asn680 homozygotes in a Swedish cohort of young men among others exhibited lower serum FSH and higher total testosterone concentrations, as well as larger testis volume and higher sperm count when compared with heterozygotes and Ala307-Ser680 homozygote carriers ${ }^{39}$. The discrete codon combination with homo/heterozygous variation of the Thr307Ala and Asn680Ser FSHR gene polymorphisms has also been reported in other Asian (Japan and China) and non-Asian (i.e. Italian, German, Egyptian, Estonian, Turkish, Iranian and Brazilian populations) patient groups $^{8,11-13,28-31,33-35,37,38}$. A synergistic effect of FSHR promoter polymorphic region $(2039 \mathrm{~A}>\mathrm{G})$ and other genetic loci (e.g., FSHB-211G >T) with Thr307Ala/ Asn680Ser allelic variants has also been reported ${ }^{7,36,40}$.

\section{Conclusion}

Our study revealed the increased risk of male infertility among the Asn/Ser (vs. Ser/Ser or Ser/Ser + Asn/Asn) genotype carriers in the group of infertile men as a whole or when subgrouping them according to various degrees of spermatogenetic impairment. Further studies on a larger population and haplotypebased association analysis instead of single SNP testing are required to confirm our findings in a more definitive fashion.

\section{Ackknowedgment}

This work was supported by the Scientific Center of Excellence for Regenerative and Reproductive Medicine (CERRM) established in 2013 at the School of Medicine, University of Zagreb, by decision of the Ministry of Science, Education and Sports, Republic of Croatia.

\section{References}

1. Desai SS, Roy BS, Mahale SD. Mutations and polymorphisms in FSH receptor: functional implications in human reproduction. Reproduction. 2013;146:235-48. https://doi: 10.1530/ REP-13-0351.

2. Simoni M, Casarini L. Mechanisms in endocrinology: genetics of FSH action: a 2014-and-beyond view. Eur J Endocrinol. 2014;170:91-107. https://doi: 10.1530/EJE-13-0624.

3. Minegishi T, Nakamura K, Takakura Y, Ibuki Y, Igarashi M, Minegishi T. Cloning and sequencing of human FSH receptor cDNA. Biochem Biophys Res Commun. 1991;175:1125-30. https://doi.org/10.1016/0006-291X(91)91682-3
4. Gromoll J, Simoni M. Genetic complexity of FSH receptor function. Trends Endocrinol Metab. 2005;16:368-73. https: //10.1016j.tem.2005.05.011.

5. Wunsch A, Ahda Y, Banaz-Yaşar F, Sontag B, Nieschlag E, Simoni M, et al. Single-nucleotide polymorphisms in the promoter region influence the expression of the human folliclestimulating hormone receptor. Fertil Steril. 2005;84:446-53. https://doi.org/10.1016/j.fertnstert.2005.02.031

6. Asatiani K, Gromoll J, Eckardstein SV, Zitzmann M, Nieschlag E, Simoni M. Distribution and function of FSH receptor genetic variants in normal men. Andrologia. 2002;34:172-6. https://doi.org/10.1046/j.1439-0272.2002.00493.x

7. Wu Q,Zhang J, Zhu P, Jiang W, Liu S, Ni M, et al. The susceptibility of FSHB $-211 \mathrm{G}>\mathrm{T}$ and FSHR G-29A, 919A $>\mathrm{G}$, 2039A $>\mathrm{G}$ polymorphisms to men infertility: an association study and meta-analysis. BMC Med Genet. 2017;18:81. https: // doi: 10.1186/s12881-017-0441-4.

8. Lend AK, Belousova A, Haller-Kikkatalo K, Punab M, Poolamets $\mathrm{O}$, Peters $\mathrm{M}$, et al. Follicle-stimulating hormone receptor gene haplotypes and male infertility in Estonian population and meta-analysis. Syst Biol Reprod Med. 2010;56:84-90. https://doi: 10.3109/19396360903456676.

9. Wu W, Cai H, Sun H, Lu J, Zhao D, Qin Y, et al. Follicle stimulating hormone receptor $\mathrm{G}-29 \mathrm{~A}, 919 \mathrm{~A}>\mathrm{G}, 2039 \mathrm{~A}>\mathrm{G}$ polymorphisms and the risk of male infertility: a meta-analysis. Gene. 2012;505:388-92. https://doi: 10.1016/j.gene.2012.02.023.

10. Tüttelmann F, Rajpert-De Meyts E, Nieschlag E, Simoni M. Gene polymorphisms and male infertility - a meta-analysis and literature review. Reprod Biomed Online. 2007;15:643-58. https://doi:10.1016/s1472-6483(10)60531-7.

11. Wu XQ, Xu SM, Wang YQ, Li Q, Wang ZQ, Zhang CL, et al. FSHR gene Thr307Ala and Asn680Ser polymorphisms in infertile men: an association study in north China and metaanalysis. Genet Mol Res. 2015;14:5592-601. https://doi: 10. 4238/2015.

12. Grigorova M, Punab M, Poolamets O, Sõber S, Vihljajev V, Žilaitienė B, et al. Study in 1790 Baltic men: FSHR Asn680Ser polymorphism affects total testes volume. Andrology. 2013; 1:293-300. https://doi: 10.1111/j.2047-2927.2012.00028.

13. Zalata AA, Hassan AH, Nada HA, Bragais FM, Agarwal A, Mostafa T. Follicle-stimulating hormone receptor polymorphism and seminal anti-müllerian hormone in fertile and infertile men. Andrologia. 2008;40:392-7. https://doi: 10.1111/ j.1439-0272.

14. Tsitlakidis D, Katopodi T, Goulis DG, Papadimas I, Kritis A. Association of follicle-stimulating hormone receptor single nucleotide polymorphisms with fertility in Greek men. J Endocrinol Invest. 2017;40:721-6. https://doi: 10.1007/s40618017-0637-7.

15. Kuijper EA, Blankenstein MA, Luttikhof LJ, Roek SJ, Overbeek A, Hompes PG, et al. Frequency distribution of polymorphisms in the FSH receptor gene in infertility patients of different ethnicity. Reprod BioMed Online. 2011;22 Supp1 1:S60-5. https://doi: 10.1016/S1472-6483(11)60010-2. 
16. Lalioti MD. Impact of follicle stimulating hormone receptor variants in fertility. Curr Opin Obstet Gynecol. 2011;23:15867. https://doi: 10.1097/GCO.0b013e3283455288.

17. Gashi Z, Elezaj S, Zeqiraj A, Grabanica D, Gashi F. Folliclestimulating hormone receptor gene polymorphism in Albanian women. Arch Med Sci Civiliz Dis. 2016;1:87-97. https://doi. org/10.5114/amscd.2016.62449.

18. Schlatt S, Ehmcke J. Regulation of spermatogenesis: an evolutionary biologist's perspective. Semin Cell Dev Biol. 2014;29: 2-16. https://doi: 10.1016/j.semcdb.2014.03.007.

19. O'Shaughnessy PJ. Hormonal control of germ cell development and spermatogenesis. Semin Cell Dev Biol. 2014;29: 55-65. https://doi: 10.1016/j.semcdb.2014.02.010.

20. Bergmann M, Behre HM, Nieschlag E. Serum FSH and testicular morphology in male infertility. Clin Endocrinol (Oxf). 1994;40:133-6. https://doi:10.1111/j.1365-2265.1994. tb02455.x.

21. Subhan F, Tahir F, Ahmad R, Khan ZD. Oligospermia and its relation with hormonal profile. J Pak Med Assoc. 1995;45: 246-7.

22. Reyes-Fuentes A, Chavarría ME, Aguilera G, Rosado A, Iranmanesh A, Veldhuis JD. Deconvolution analysis of bioassayable LH secretion and half-life in men with idiopathic oligoasthenospermia. Int J Androl. 1997;20:118-25. https:// doi.org/ 10.1046/j.1365-2605.1997.00104.x

23. Buvat J. Hyperprolactinemia and sexual function in men: a short review. Int J Impot Res. 2003;15:373-7. https://doi: 10.1038/sj.ijir.3901043.

24. Masud S, Mehboob F, Bappi MU. Severe hyperprolactinemia directly depresses the gonadal activity causing infertility. Esculapio J Services Inst Med Sci. 2007;2:25-7. https://doi: 10.4103/0974-1208.86094

25. Soler Fernández JM, Caravaca Magariños F, Domínguez Bravo C, Murillo Mirat J, Aparicio Palomino A, Herrera Puerto J. Correlation of serum prolactin, sperm count and motility. Prevalence of hyperprolactinemia in the infertile male. Arch Esp Urol. 1990;43:891-5. https://www.ncbi.nlm.nih.gov/pubmed/ 2291683\#

26. Perez Mayorga M, Gromoll J, Behre HM, Gassner C, Nieschlag E, Simoni M. Ovarian response to follicle-stimulating hormone (FSH) stimulation depends on the FSH receptor genotype. J Clin Endocrinol Metab. 2000;85:3365-9. https:// doi:10.1210/jcem.85.9.6789

27. Foresta C, Selice R, Garolla A, Ferlin A. Follicle-stimulating hormone treatment of male infertility. Curr Opin Urol. 2008; 18:602-7. https://doi: 10.1097/MOU.0b013e328313647d.

28. Safarinejad MR, Shafiei N, Safarinejad S. Evaluating the role of the FSH receptor gene Thr307-Ala and Asn680-Ser polymorphisms in male infertility and their association with semen quality and reproductive hormones. BJU Int. 2011;108:117-25. https://doi: 10.1111/j.1464-410X.2010.09890.

29. Balkan M, Gedik A, Akkoc H, Izci Ay O, Erdal ME, Isi H, et al. FSHR single nucleotide polymorphism frequencies in proven fathers and infertile men in southeast Turkey. J Biomed Biotechnol.2010;2010:640318.https://doi: 10.1155/2010/640318.
30. Shimoda C, Koh E, Yamamoto K, Matsui F, Sugimoto K, Sin $\mathrm{HS}$, et al. Single nucleotide polymorphism analysis of the follicle-stimulating hormone (FSH) receptor in Japanese with male infertility: identification of codon combination with heterozygous variations of the two discrete FSH receptor gene. Endocr J. 2009;56:859-65. https://doi:10.1507/endocrj.k09e-130.

31. Simoni M, Gromoll J, Höppner W, Kamischke A, Krafft T, Stähle D, et al. Mutational analysis of the follicle-stimulating hormone (FSH) receptor in normal and infertile men: identification and characterization of two discrete FSH receptor isoforms. J Clin Endocrinol Metab. 1999;84:751-5. https:// doi:10.1210/jcem.84.2.5500.

32. Ferlin A, Pengo M, Selice R, Salmaso L, Garolla A, Foresta C. Analysis of single nucleotide polymorphisms of FSH receptor gene suggests association with testicular cancer susceptibility. Endocr Relat Cancer. 2008;15:429-37. https://doi: 10.1677/ ERC-07-0257.

33. Ahda Y, Gromoll J, Wunsch A, Asatiani K, Zitzmann M, Nieschlag E, et al. Follicle-stimulating hormone receptor gene haplotype distribution in normozoospermic and azoospermic men. J Androl. 2005;26:494-9. https://doi:10.2164/jandrol. 04186.

34. Pengo M, Ferlin A, Arredi B, Ganz F, Selice R, Garolla A, et al. FSH receptor gene polymorphisms in fertile and infertile Italian men. Reprod Biomed Online. 2006;13:795-800. https:// doi.org/10.1016/S1472-6483(10)61026-7.

35. Gharesi-Fard B, Ghasemi Z, Shakeri S, Behdin S, Aghaei F, Malek-Hosseini Z. The frequency of follicle stimulating hormone receptor gene polymorphisms in Iranian infertile men with azoospermia. Iran J Reprod Med. 2015;13:673-8. https:// www.ncbi.nlm.nih.gov/pmc/articles/PMC4695681/

36. Tüttelmann F, Laan M, Grigorova M, Punab M, Sõber S, Gromoll J. Combined effects of the variants FSHB $-211 \mathrm{G}>\mathrm{T}$ and FSHR 2039A $>\mathrm{G}$ on male reproductive parameters. J Clin Endocrinol Metab. 2012;97:3639-47. https://doi: 10.1210/ jc.2012-1761.

37. Ghirelli-Filho M, Peluso C, Christofolini DM, Gava MM, Glina S, Barbosa CP, et al. Variants in follicle-stimulating hormone receptor gene in infertile Brazilian men and the correlation to FSH serum levels and sperm count. Reprod Sci. 2012;19:733-9. https://doi: 10.1177/1933719111432872.

38. Li Y, Gu A, Yang H, Ding X, Ji G, Lu C, et al. FSH receptor gene polymorphisms in fertile and infertile Han-Chinese males. Clin Chim Acta. 2011;412:1048-52. https://doi: 10. 1016/j.cca.2011.02.016.

39. Lindgren I, Giwercman A, Axelsson J, Lundberg Giwercman Y. Association between follicle-stimulating hormone receptor polymorphisms and reproductive parameters in young men from the general population. Pharmacogenet Genomics. 2012;22:667-72.https://doi:10.1097/FPC.0b013e3283566c42.

40. Grigorova M, Punab M, Punab AM, Poolamets O, Vihljajev V, Zilaitienė $\mathrm{B}$, et al. Reproductive physiology in young men is cumulatively affected by $\mathrm{FSH}$-action modulating genetic variants: FSHR -29G/A and c.2039 A/G, FSHB -211G/T. PLoS One. 2014;9:e94244. https://doi: 10.1371/journal.pone.0094244. 
Sažetak

\title{
POJAVNOST GENSKOG POLIMORFIZMA RECEPTORA 2039A>G FOLIKULARNO STIMULIRAJUĆEG HORMONA I RIZIK MUŠKE NEPLODNOSTI U ALBANSKOJ POPULACIJI
}

\author{
E. Shkelzen, F. Paic, F. Stipoljev, Z. Gashi, A. Zeqiraj, A. Lila i T. Nikuševa Martić
}

Cilj ovoga istraživanja bio je odrediti pojavnost alela i varijante genotipa receptora folikularno stimulirajućeg hormona (FSHR) na poziciji Asn680Ser kod muškaraca albanske populacije u odnosu na kliničke parametre neplodnosti. Istraživanje je obuhvatilo 114 neplodnih muškaraca (srednja dob 35,04 $\pm 5,85$ godina) svrstanih prema razini oštećenja spermiograma (oligoastenozoospermija, astenozoospermija i normospermija) te 112 plodnih muškaraca (srednja dob 36,44 \pm 7,05 godina) s urednim nalazom spermiograma. Genotipizacija gena FSHR na poziciji 680 učinjena je primjenom TaqMan probe. Kod svih sudionika istraživanja učinjena je analiza sjemena i reprodukcijskih hormona uključujući FSH, luteinizirajući hormon, prolaktin i testosteron. U kontrolnoj skupini ispitanika kod FSHR Asn680Ser genotipa utvrđena pojavnost Asn/Ser bila je 42\%, Ser/Ser 33,9\% i Asn/Asn 24,1\%, dok se u skupini neplodnih ispitanika incidencija kretala od 56,1\% za Asn/Ser, 22,8\% za Ser/Ser i $21,1 \%$ za Asn/Asn ( $\chi^{2}$-test; p=0,08). Nije ustanovljena značajna statistička povezanost između razine hormona, karakteristika sjemena, stanja plodnosti u varijanti gena FSHR Asn680Ser u kontrolnoj skupini u odnosu na ispitanike u skupini neplodnih muškaraca. Ipak, primjenom prilagođene, logističke i regresijske analize (dob, indeks tjelesne mase, pušenje i alkohol kao kovarijable) utvrđeno je da postoji veća vjerojatnost javljanja muške neplodnosti kod nositelja heterozigota Asn/Ser koji su povezani sa sniženim vrijednostima parametara sjemena (morfologija, koncentracija, ukupan broj i pokretljivost). Zaključno možemo utvrditi da ovo istraživanje potvrđuje ranija izvješća kako ne postoji značajna povezanost između polimorfizma FSHR Asn680Ser i muške neplodnosti. Ipak, navedeni podaci upućuju na to da Asn/Ser genotip može povisiti rizik muške neplodnosti u albanskoj populaciji.

Ključne riječi: Neplodnost muškarca; Prolaktin; Testosteron; Receptori, FSH; Studije slučajeva i kontrola 